\section{Junge Allergologen legen los}

Frisches Blut will die Deutsche Gesellschaft für Allergologie und klinische Immunologie und das bekommt sie: 18 rege Nachwuchsallergologen trafen sich zur Gründung der „Junior Members“ am 10. März 2011 in Mainz.

- in in ganz besonderer Programmpunkt des 23. Mainzer-Allergieworkshops war das Gründungstreffen der „Junior Members" der Deutschen Gesellschaft für Allergologie und klinische Immunologie (DGAKI). Prof. Dr. Eckard Hamelmann aus Bochum moderierte das Ereignis an: „Die Allergologie ist elementar bedroht dadurch, dass wir zu wenig aktiven Nachwuchs haben." Trotz toller Forschung leide gerade die klinische Ausbildung abwechselnd unter Stellen- und dann wieder unter Bewerbermangel. „Wir wollen bewusst die jungen Leute ansprechen, denn sie müssen das hier alles einmal übernehmen“, fügte er hinzu. Achtzehn junge Wissenschaftler und Kliniker trauten sich das zu: darunter 13 Grundlagenforscher, drei Dermatologen, eine Pädiaterin und eine Ökotrophologin. Pneumologen, Hals-Nasen-OhrenSpezialisten wie auch Innere Mediziner fehlen noch und sind aufgerufen, sich ebenfalls zu beteiligen.

Die Junior Members sollen innerhalb der DGAKI eine eigene Sektion bilden. Aber wie konstituiert sich eine
Sektion eigentlich? Zuerst wählten die in Mainz zusammengekommenen Junioren ihre erste Sprecherin: die Dermatologin Dr. Margarete Niebuhr stellt sich der spannenden Aufgabe. Ihre Stellvertreterin ist die Biologin Dr. Melanie Albrecht. Beide forschen an der Medizinischen Hochschule Hannover.

Jetzt ging es darum, die Sektion inhaltlich zu strukturieren. Dazu sollten als nächstes gemeinsame Ziele formuliert werden. Schnell war klar, dass es eine eigene Postersession während des Deutschen Allergiekongresses im September in Wiesbaden geben soll - die besten Poster werden sogar prämiert. Ab sofort können dazu Abstracts unter der Internetadresse www.allergiekongress.de online eingereicht werden. Als Junior Member gibt man sich durch Setzen eines Kreuzes beim entsprechenden Eingabefeld zu erkennen.

Wie es weitergeht: Sektionstreffen beim Mainzer-Allergie-Workshop sind angedacht. Die Fortbildung der jungen Allergologen will programmatisch gestaltet werden: Es sollen interne Workshops mit eingeladenen Referenten aus Klinik,

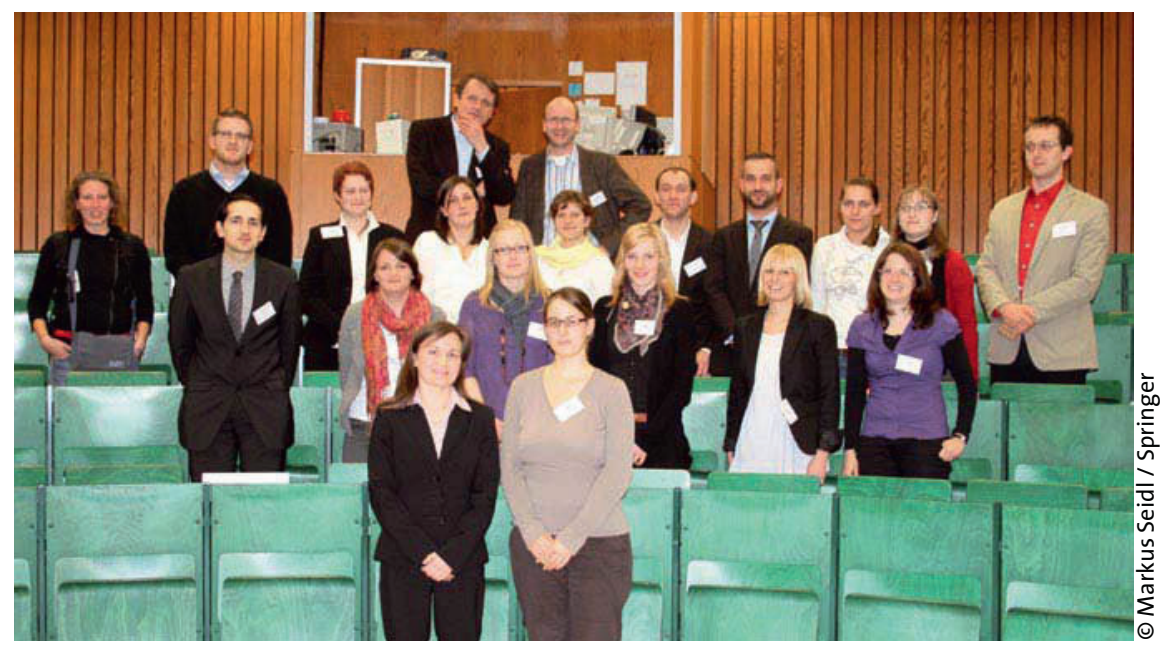

Die Gründungsmitglieder - das Herzstück - der Junior Members der DGAKI stellten sich mit ihrer ersten Sprecherin Dr. Margarete Niebuhr (vorn links) und deren Stellvertreterin Dr. Melanie Albrecht (rechts) vor. Im Hintergrund lauschten Prof. Dr. Thilo Jakob (links) und Moderator Prof. Dr. Eckard Hamelmann (rechts) den ersten Takten.

Jetzt Junior Member werden

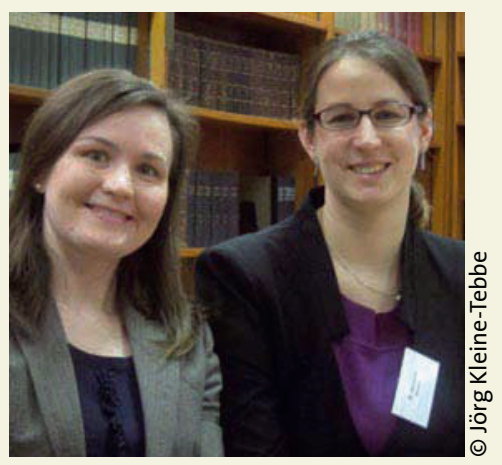

Jedes Mitglied der DGAKI bis zum Alter von 35 Jahren ist ein Junior Member damit sind folgende Vorteile verbunden:

— Beitragsfreie Mitgliedschaft

- Neun Ausgaben des Allergo Journal pro Jahr

— Kostenfreie Registrierung für den

Deutschen Allergiekongress bei ak-

tiver Teilnahme (Vortrag oder Poster)

_ Austausch mit jungen Kollegen und

mit Fachexperten

_ Einblick in die Arbeit der Fachsektionen

Weitere Informationen gibt es unter: www.dgaki.de/sektionenags/

sektionen/junior-members

translationaler Forschung und Industrie stattfinden. Weit oben stehen auch Karriereplanung und das Einwerben von Drittmitteln auf dem Themenplan. Ein eigener Bereich auf der Webseite der DGAKI soll daneben den gegenseitigen Austausch der Junior Members fördern und es erleichten, Ideen zu äußern und zu erproben. Verena Raker von der Universitäts-Hautklinik Mainz stellte sich als Web-Betreuerin zur Verfügung.

Ideelle Unterstützung finden die Junior Members in den einzelnen Fachsektionen, an deren Sitzungen sie entsprechend ihren speziellen Interessenlagen nicht nur aktiv teilnehmen können, sondern sogar sollen. Auch Hamelmann wird beratend zur Seite stehen. Wer sich bei den Junior Members der DGAKI engagieren möchte, kann per E-Mail an niebuhr.margarete@mh-hannover.de Kontakt aufnehmen. Die neue Sektion vertritt Mitglieder, die nicht älter als 35 Jahre sind. Bis zu diesem Alter ist die Mitgliedschaft bei der DGAKI übrigens beitragsfrei. 